\title{
On subjective impressions or how to put numbers to visual illusions
}

\author{
J.A. Martín-Pereda, A. González-Marcos
}

E.T.S. Ingenieros de Telecomunicacion. Universidad Politecnica de Madrid, Madrid, Spain

\begin{abstract}
This paper presents a simple theory to analyze some of the more common visual illusions. The reported mathematical model is able to give some numerical results concerning well known phenomena as the Zollner, Muller-Lyer or Wundt illusions. We present two different approaches, the first one related with the symmetrical operations present in each image and the second one concerning a proposed vector field related with the lines present at the image.
\end{abstract}

Keywords - Visual illusions, subjective image properties.

\section{INTRODUCTION}

To extract information about object dimensions or shapes is one of the main tasks for every image processor working nowadays. There is a clear reason to that function. The obtained information is employed in different applications ranging from objects classification to pattern detection. Any one of these applications is related with objective properties. Their results are employed in situations where there is a need to obtain absolute values related with real data. Nevertheless, there is another group of situations where the obtained results should be in agreement with subjective impressions felt by human beings.

One of the concepts more difficult to handle is the one related with Visual Perception. Visual Perception is the process of acquiring knowledge about object characteristics by extracting information from the relation between this object and its boundary conditions - other objects near to it, relative position, and so on. In this way, Visual Perception concerns the acquisition of knowledge and is distinct from purely optical processes such as photographic ones. These processes lack of perceptual capabilities and they do not know anything about the scenes they record. Hence, the knowledge achieved by visual perception concerns objects and events in their environment. An object, as seen by a living body, is not just a simple object. It is an object in a certain place and with some particular conditions. If place or conditions change, the perception of such an object may change.

Perhaps the most famous of all distortions is the doubleheaded-arrow figure devised by Franz Müller-Lyer (Fig. 1). This figure is so simple that it was immediately accepted as a primary target for theory and experiment. The influence of angles is clear with respect to their influence on the perception of length. In almost everyone of the reported interpretation of the illusion, there is a common factor. It is the importance of angles in determining sizes and parallelism. This fact appears clearly in the Hering (Fig. 2.a) and Wundt (Fig. 2.b) illusions. Effects are slightly different in every one of them. But a lack of parallelism is the

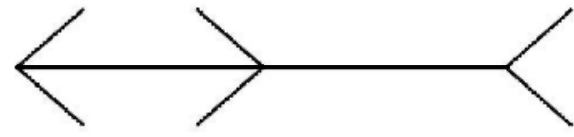

(a)
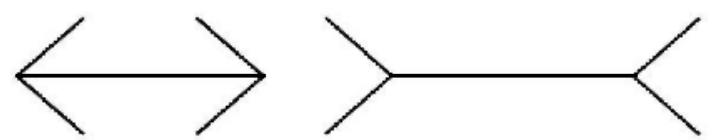

(b)

Fig. 1. - Muller-Lyer Illusion.

common "feeling". In Wundt illusion, lines tend to diverge at their ends. On the contrary, Hering effect "expands" the central part. These effects are strongly dependent of the crossing lines characteristics: angle, distance between them, thickness and color. Angles are, in any case, the most important factor to determine the effect strength.

A theory to interpret these facts will be reported in this paper. First, we will establish some general concepts, most of them based on previous works by us [1]-[2], and from them we will develop some principles in order to quantify illusions. The theory to be reported here is just a first approach to a more general one
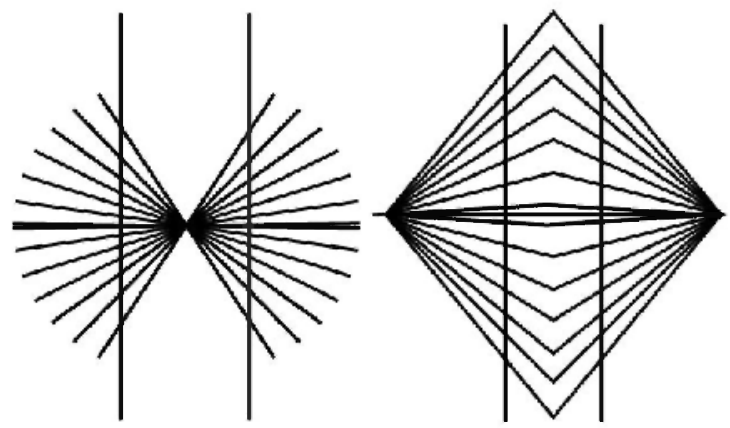

Fig. 2 - (a) Hering and (b) Wundt illusions 


\section{ANALYSIS OF THE ZÖLLNER ILLUSION}

The Zollner illusion is related with the lack of parallelism "seen" when lines with a certain tilt intersect two parallel lines (Fig. 3). In order to analyze this effect we will

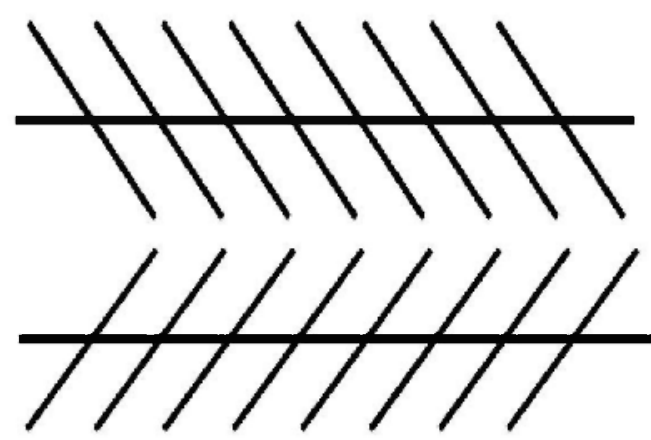

F1g. 3 - Zollner 1llusion

use a small part of this figure (Fig. 4) because the more important facts, namely symmetry operations, appear in the last one. We will use in this effect the concept of visual oriented density. We define it as the "distributed weight" of a certain detail of the image with respect to the preferential line in the subject. The preferential line is, in this case, the twofold symmetry axis. The distributed weight corresponds to the columns in the physiological model that have been excited in each partial detail of the image seen by the observer. In the case shown in Fig. 4 just two different

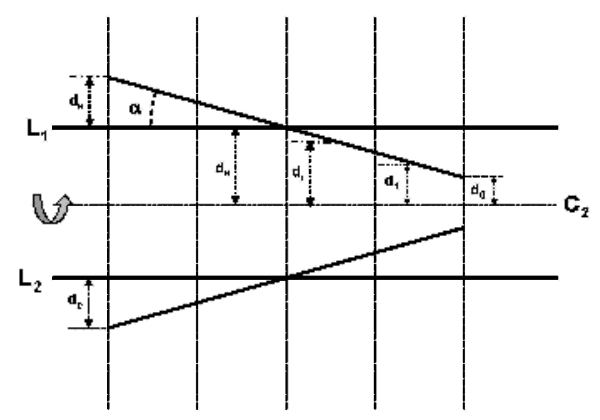

Fig. 4.- Main parameters in the Zollner illusion.

columns will be excited because there just two different line orientations. Another point needs now to be considered. Parallelism has imposed the central line as symmetry reference. Hence, the weight of parallel lines must be different to the weight of the crossing lines. According to experimental results, these crossing lines impose a different influence depending on their angle values. Stronger effects correspond to angles between $30^{\circ}$ and $50^{\circ}$. For example, $85^{\circ}$ or $5^{\circ}$ angles do not have almost any effect on parallel illusions.

To analyze this situation we have divided it in four columns. This indicates our assumption that, in this case, only four cortical orientation columns may be excited. In each one of them, two orientation bands detect two different orientations. We call $2 \mathrm{~d}_{\mathrm{e}}$ the distance between parallel lines, $\alpha$ the angle of the crossing lines with the parallel ones and $d_{i}$ the distance from the middle point of each segment in a column to the twofold axis of symmetry. With these data, we propose the following model to calculate the parallelism impression. We establish the following matrix as a main indication to parallelism

$$
\pi_{\beta}=\left[\pi_{\alpha 1}-\pi_{\alpha 2}, \pi_{\alpha 3}-\pi_{\alpha 4}, \ldots\right]
$$

Elements in this matrix are given by

$$
\pi_{\alpha k}=\sigma_{\alpha k} / \wp_{\alpha k}
$$

with

$$
\sigma_{\alpha k}=\left\{\sum_{i=1}^{n} \omega_{i} \delta_{i}\right]_{\alpha k}+S\left\{\sum_{j=1}^{n} \omega_{j} \delta_{j}\right\}_{\alpha l}
$$

where $\omega_{\mathrm{i}}$ is the "weight" given to the $\mathrm{i}$-th element and $\delta_{\mathrm{i}}$ its distance to the central line (symmetry axis). $\mathbf{S}$ is the symmetry operation imposed to the object, in our case, the twofold axis. This symmetry operation carries one of the details of the object to the other one. $\sigma_{\alpha k}$ is a measure of the details that have "impressed" to sensorial columns. $\wp$ is a similar effect corresponding to the parallel lines.

Elements in matrix $\pi$ are zero when parallelism is "seen". The difference with zero gives an idea about how the degree of parallelism is "not seen". A simple example may give a more simple interpretation of this model.

If we assume the following values for above indicated parameters:

$$
\begin{gathered}
\delta_{\mathrm{e}}=6 \quad \delta_{1}=3 \quad \delta_{1}=3+2(\mathrm{i}-1) \\
\omega=0.5
\end{gathered}
$$

Every "weight" has the same value in this case, because the considered detail is a segment.

$$
\wp_{\mathrm{i}}=6
$$

Columns are numbered from right to left.

With these data, matrixes corresponding to elements above and below the central line are

$$
\begin{gathered}
\sigma_{1}=\{6 \times 1+9 \times 0.5 ; 6 \times 1+7 \times 0.5 ; 6 \times 1+5 \times 0.5 \\
6 \times 1+3 \times 0.5\} \\
\sigma_{2}=\{6 \times 1+9 \times 0.5 ; 6 \times 1+7 \times 0.5 ; 6 \times 1+5 \times 0.5 \\
6 \times 1+3 \times 0.5\}
\end{gathered}
$$




$$
\begin{gathered}
\sigma_{\mathrm{T}}: \quad \sigma_{1}+\mathrm{C}_{2} \sigma_{2} \\
=\{10.5+10.5 ; 9.5+9.5 ; 8.5+8.5 ; 7.5+7.5\}= \\
=\{21 ; 19 ; 17 ; 15\}
\end{gathered}
$$

Hence

$$
\begin{aligned}
& \wp_{\mathrm{T}}: \quad \wp_{1}+\mathrm{C}_{2} \wp_{2} \\
& =\{6+6 ; 6+6 ; 6+6 ; 6+6\}= \\
& =\{12 ; 12 ; 12 ; 12\}
\end{aligned}
$$

And so

$$
\pi_{\mathrm{T}}=\left(\wp_{\mathrm{T}} / \sigma_{\mathrm{T}}\right): \quad=\{0.57 ; 0.63 ; 0.70 ; 0.8\}
$$

These numbers indicate the existence of a certain "lack of parallelism" in the obtained impression. If we subtract now to the number at a column the corresponding to the adjacent column in the right, we get

$$
\left\{\pi_{\mathrm{T} 1}\right\}=\left\{\pi_{\mathrm{T} 1}-\pi_{\mathrm{T}(1-1)}\right\}: \quad=\{0.06 ; 0.07 ; 0.1\}
$$

and by successive repeating of this operation

$$
\begin{gathered}
\left\{\pi_{\mathrm{T} 2}\right\}=\left\{\pi_{\mathrm{T} 1 \mathrm{i}}-\pi_{\mathrm{T} 1(\mathrm{I}-1)}\right\}:=\{0.01 ; 0.03\} \\
\left.\left\{\pi_{\mathrm{T} 3}\right\}=\left\{\pi_{\mathrm{T} 2 \mathrm{i}}-\pi \pi_{\mathrm{T} 2(1-1)}\right\}:=\mathbf{0 . 0 2}\right\}
\end{gathered}
$$

This number gives an idea about the discrepancy between the real structure of the object and the perceived configuration. This example may give a closer idea about our model if we compare it with the obtained result from

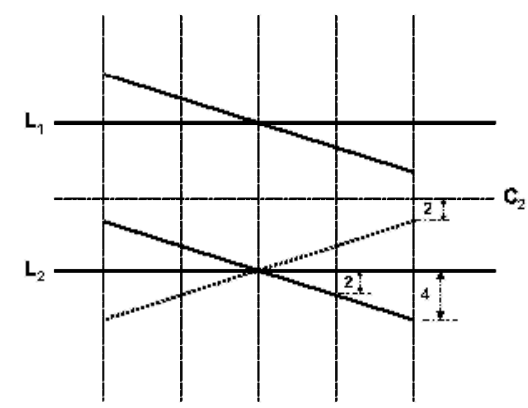

Fig. 5.- Symmetry operations in the case of equal angle crossing lines.

structure in Fig. 6. In this case, both crossing lines intersect the parallel segments with equal angles. It is because that the previous twofold symmetry axis no longer remains. If a $\mathrm{C}_{2}$ rotation is performed to line $\mathrm{L}_{1}$, the new position is the indicated by a segmented line. In any case, because with respect to the parallel lines, this axis remains a $C_{2}$ axis, this is the symmetry operation we have to apply. With similar numbers that in the previous case, the result is now:

$$
\begin{aligned}
& \sigma_{1}=\{6 \times 1+9 \times 0.5 ; 6 \times 1+7 \times 0.5 ; 6 \times 1+5 \times 0.5 ; 6 \times 1+3 \times 0.5\} \\
& \sigma_{2}=\{6 \times 1+3 \times 0.5 ; 6 \times 1+5 \times 0.5 ; 6 \times 1+7 \times 0.5 ; 6 \times 1+9 \times 0.5\}
\end{aligned}
$$

$\sigma_{\mathrm{T}}: \sigma_{1}+C_{2} \sigma_{2}=\{10.5+7.5 ; 9.5+8.5 ; 8.5+9.5 ; 7.5+10.5\}=$
And so

$$
=\{18 ; 18 ; 18 ; 18\}
$$

$$
\pi_{\mathrm{T}}=\left(\wp_{\mathrm{T}} / \sigma_{\mathrm{T}}\right): \quad=\{0.67 ; 0.67 ; 0.67 ; 0.67\}
$$

Hence

$$
\left\{\pi_{\mathrm{T} 1}\right\}=\left\{\pi_{\mathrm{T} 1}-\pi_{\mathrm{T}\{1-1)}\right\}: \quad=\{\mathbf{0 . 0} ; \mathbf{0 . 0} ; \mathbf{0 . 0}\}
$$

This result indicates that the feeling obtained by the observer is parallelism. Lines crossing with similar angle do not change the impression "seen" by the observer.

From above facts, it is clear that the present method gives some type of objective information about the subjective impression got by any observer. The method may be easily generalized

\section{HERINGS AND WUNDTS ILLUSIONS}

The main difference between this case (Fig. 2) and the previously reported one is that angles crossing the parallel lines are now different at each position. In order to solve this situation we introduce a certain "vector field". This vector field, with a discrete character, originates by the addition of

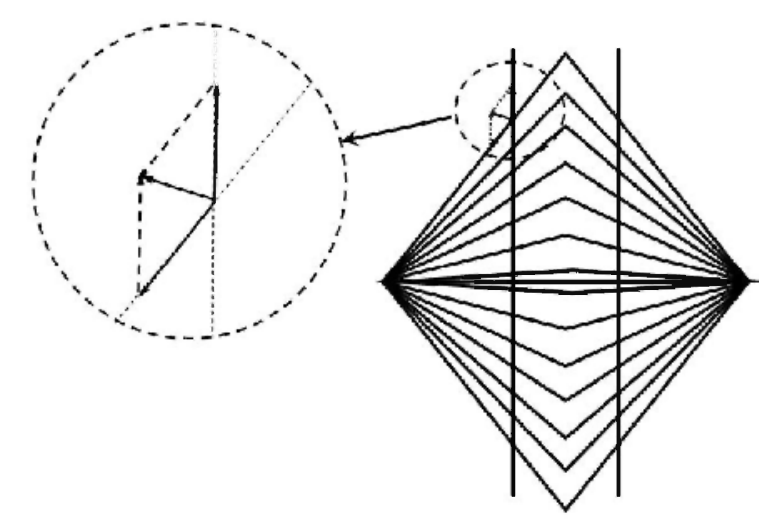

Fig. 6 vector field detcmination in the Fund illusion

two vectors at each one of the intersecting points between parallel and crossing lines. These vectors appear at the inset of Fig. 6. Vectors corresponding to the parallel lines have a conventional magnitude of 1 unit and have the direction of these lines. On the contrary, vector at the crossing lines, with same direction of them, have a magnitude depending on the angle. This value is obtained by multiplying the unity by a certain weight. This weight corresponds to the quantitative measurements of some illusions given by R. L. Gregory. Hence, each point in the intersection of parallel and crossing lines has a vector associated with it. This is the above indicated "discrete vector field".

We obtain a graphical representation of the vector field by performing this operation to every intersection point in the Wundt illusion. The final result appears in Fig. 7 (a)(c). Fig. 7 (a) gives the obtained vector field. At Fig. 7 (b) just 
the resulting vectors appear as well as the parallel lines. In Fig. 7 (c) the two parallel lines have disappeared and the vector ends are joined. We obtain a line with a certain curvature. These lines are similar to the virtually present in the virtual illusion.

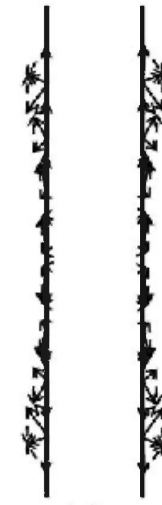

(a)

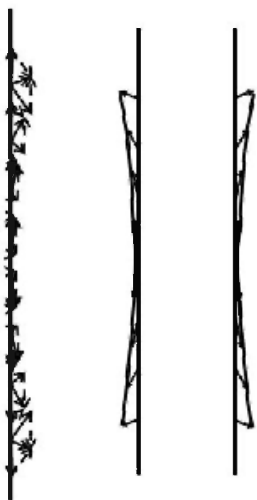

(b)

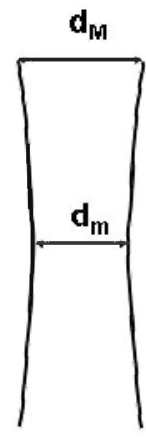

(c)
Fig. 7. Vector field for Wundt illusion and resulting final lines

In this case, two are the main parameters to be considered. They are the "minimum" and "maximum" distances appearing between the two lines, $\mathrm{d}_{\mathrm{m}}$ and $\mathrm{d}_{\mathrm{M}}$. If we considerer $\mathrm{d}_{\mathrm{m}}$ as the "real" distance between parallel lines, we may define a quantity, $\check{\zeta}$, given by

$$
\xi=\left(d_{M}-d_{m}\right) / d_{m}
$$

as a measure of the "curvature" degree. When its value is zero, the feeling is of a lack of curvature and, as large is the obtained number, larger is the perceived sensation.

A similar method may be applied to the Hering illusion.

\section{CONCLUSIONS}

Zollner and Wundt illusions have been studied and a simple model to justify some of their characteristics has been reported. This work is just a first proposal to analyze visual illusions. Wundt and Zollner illusions have been initiated in this paper and Müller-Lyer was studied by us in a previous one. The employed methods have in the three cases some common points but there is yet a need to integrate them in a more general theory. The cases presented need the introduction of some new concepts. For example, the influence of the concept that we have called "subjective weight" or "visual density" has not been extensively employed in the present paper. But the proof that it is possible to "put numbers to visual illusions" has been clarified in this work. A final point should be indicated. In this paper a certain global approach to study visual illusions has been presented. But some more practical aspects, related with the way visual signals are processed and related, have to be introduced. These facts have to be related with the way signals go from retina to visual cortex. If a system with similar properties to some visual systems in living bodies is to be developed, similar type of signals should be employed. This approach has been reported by us in previous papers [1]-[2] and should be connected with the present one.

\section{ACKNOWLEDGMENT}

This work was partly supported by CICYT, grant TIC200304309.

\section{REFERENCES}

[1] J.A. Martin-Pereda and A. Gonzalez-Marcos, "A new method to analyse the perception of size". Applications of Digital Image Processing XXIV. SPIE. Vol 4472 pp. 75-84 (2001)

[2] J.A. Martin-Pereda \& A. Gonzalez-Marcos, "Extraction of subjective properties in image processing", Photonic Devices and Algorithms for Computing IV. SPIE vol. 478817. pp: 73-82. (2002)

[3] J.A. Martin-Pereda \& A. Gonzalez-Marcos, "A method to study the perception of subjective image characteristics" 2nd European Medical and Biological Engineering Conference EMBEC'02 Proc. pp. 1-864-865. (2002). 